\title{
Coprime Conjugacy Class Sizes
}

\author{
A.R.Camina \& R.D.Camina \\ School of Mathematics, University of East Anglia, \\ Norwich, NR4 7TJ, UK; a.camina@uea.ac.uk \\ Fitzwilliam College, Cambridge, CB3 0DG, UK; \\ rdc26@dpmms.cam.ac.uk
}

\section{Introduction}

In this paper we consider the influence of conjugacy class sizes on the structure of finite groups. Over the years there have been many papers on this topic and we make a contribution to this study. Although the problems are entirely group theoretic it has been realised that the language of graph theory can sometimes improve the understanding of the results and provide motivation for some problems. One result we will use and which has an interesting interpretation in graph theory language is due to Kazarin from 1981 [17]. He discussed groups with isolated conjugacy classes: a finite group $G$ has isolated conjugacy classes if there exist two conjugacy classes with coprime sizes $m$ and $n$ such that every conjugacy class size is coprime to either $m$ or $n$. Recall, the common divisor graph $\Gamma(X)$ of a set $X \subset \mathbb{N}$ is defined as follows. Let $X^{*}=X \backslash\{1\}$ be the vertex set of $\Gamma(X)$ (we do not assume $1 \in X$ ) and join two vertices, $x$ and $y$, if and only if they are not coprime. Now, let $X_{G}$ be the set of conjugacy class sizes of a finite group $G$. Then Kazarin's result concerns groups where the graph, $\Gamma\left(X_{G}\right)$, is disconnected or has diameter at least 3 , he classified all such groups.

In this paper we prove some results about finite groups whose graph has no triangles or, alternatively, given any three conjugacy class sizes there are two which are coprime. Our main theorem is:-

Main Theorem Let $G$ be a finite group with the property that given any three distinct conjugacy class sizes greater than 1 there is a pair which is 
coprime. Then, $G$ has at most three conjugacy class sizes greater than 1, and $G$ is soluble.

We have the following two corollaries (for proofs see the end of the paper):-

Corollary A Let $G$ be a finite group with trivial centre and with at most three distinct conjugacy class sizes greater than 1 . Then $G$ is either soluble or $\operatorname{PSL}\left(2,2^{a}\right)$.

Corollary B Let $G$ be a finite A-group with at most three distinct conjugacy class sizes greater than 1 . Then $G$ is either soluble or $\operatorname{PSL}\left(2,2^{a}\right)$. (Note an $A$-group is a group all of whose Sylow subgroups are abelian)

Fang and Zhang considered the more restrictive case when $G$ is a finite group with the property that given any three distinct non-trivial conjugacy classes there is a pair of coprime order. They classified all groups that satisfy this property [10].

Before discussing this result further we will introduce some notation. Let $n_{1}>n_{2}>n_{3} \cdots>n_{r+1}=1$ be the distinct conjugacy class sizes of a group $G$. Then Itô called $r$ the rank of $G$ and the vector $\left(n_{1}, n_{2}, \cdots, n_{r}, 1\right)$ the conjugate type vector of $G$. Note that if $x \in G$, then the order of the conjugacy class containing $x$ is given by the index of the centraliser of $x$ in $G$ and denoted by $\operatorname{Ind}_{G}(x)$.

So, the theorem above can be restated by saying that if the graph $\Gamma\left(X_{G}\right)$ is triangle-free then $G$ has rank at most three and $G$ is soluble. Note that Itô [14] showed that if $G$ has rank 1 then $G$ is nilpotent, and then Itô [15] and A.Camina [7] showed that groups of rank 2 are soluble. However, groups of rank 3 are not soluble as the example $\operatorname{PSL}\left(2,2^{a}\right)$ illustrates, in fact Itô [16] showed that these are the only simple groups of rank 3. This and our result lead one to ask whether it would be possible to give some type of classification of rank 3 groups.

In 1930 Tchounikhin [19] considered the situation when $G$ has two conjugacy class sizes which are coprime, this is equivalent to asking that the graph is not complete, and conjectured that such a group was not simple. This was finally proved by Arad and Fisman [2] using the CFSG. Tchounikhin showed that if $G$ has three conjugacy class sizes which are coprime then $G$ is not simple. We make a further contribution to this situation in this paper.

Finally we note that Beltrán and Felipe [5] classify groups which are soluble and have rank 3 and whose non-trivial conjugacy class sizes $\{a, b, c\}$ 
satisfy $\operatorname{gcd}(a, c)=1, a$ divides $b$ and $b / a$ divides $c$. This is a special case of the condition we consider in Section 3 .

\section{Bounding conjugate rank}

In this section we prove the following theorem.

Theorem 1 Let $G$ be a finite group with the property that given any three distinct conjugacy class sizes greater than 1 there is a pair which is coprime. Then, $G$ has conjugate rank at most 3.

To prove this theorem we start with a lemma, which relies on the concept of a kernel of a subset $A$ of a group $G: \operatorname{ker}(A):=\{x \in G: x A=A\}[9]$. The kernel is a subgroup of $G$ and since $A$ is a union of cosets of $\operatorname{ker}(A)$ it follows that the order of $\operatorname{ker}(A)$ divides the order of $A$. Recall, if $B$ and $C$ are conjugacy classes of coprime sizes then $B C$ is a conjugacy class, $|B C| \geq$ $\max \{|B|,|C|\}$ and $|B C|$ divides $|B||C|$. Let $X_{G}$ denote the conjugacy class sizes of $G$ and $\Gamma=\Gamma\left(X_{G}\right)$, the common divisor graph of $X_{G}$. Suppose $|B|>|C|$, then, if $|B C|>|B|$ we have a path of length 2 in $\Gamma$ from $|C|$ to $|B|$. However, if $|B C|=|B|$ we can then repeat the process with conjugacy classes $B C$ and $C^{-1}$. If again we get $\left|B C C^{-1}\right|=|B|$, note that $B \leq B C C^{-1}$ and so $B=B C C^{-1}$ and $C C^{-1} \subseteq \operatorname{ker}(B)$.

We denote the distance of two vertices $x$ and $y$ in a graph $\Gamma$ by $d_{\Gamma}(x, y)$.

Lemma 1 Let $1<a<b_{1}<b_{2}$ be pairwise coprime indices of elements of a finite group $G$. Let $X_{G}$ denote the set of conjugacy class sizes of $G$ (equivalently the set of indices of $G$ ) and let $\Gamma=\Gamma\left(X_{G}\right)$. Then there exists an $i$ such that $d_{\Gamma}\left(a, b_{i}\right)=2$ and furthermore there exists an index $c$ such that $c>b_{i}$ and $c$ divides $a b_{i}$.

Proof. Let $A, B_{1}, B_{2}$ be conjugacy classes of sizes $a, b_{1}, b_{2}$ respectively. By the previous paragraph, if there does not exist an index $c$ satisfying the conclusions of the lemma, then $A A^{-1} \subseteq \operatorname{ker}\left(B_{i}\right)$ for $i=1,2$. But $\left(\left|\operatorname{ker}\left(B_{1}\right)\right|,\left|\operatorname{ker}\left(B_{2}\right)\right|\right)=1$, yielding a contradiction. $\square$.

We note that this lemma also appears in [8] as Lemma 9, but set in the language of schemes. 


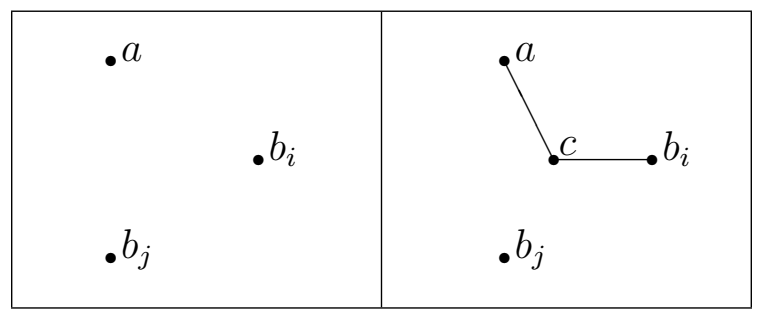

Figure 1: This illustrates the effect of Lemma 1

Proof of Theorem 1. Let $G$ satisfy the hypotheses of the theorem and $X_{G}$ denote the conjugacy class sizes of $G$, denote $\Gamma\left(X_{G}\right)$ simply by $\Gamma$. If $\Gamma$ is disconnected then $G$ is quasi-Frobenius (that is the quotient group of $G$ by its centre is Frobenius) and $G$ is of rank 2 [17]. So we can assume $\Gamma$ is connected. Kazarin has classified all groups for which the graph $\Gamma$ has diameter 3, they are a direct product of a quasi-Frobenius group and a nonabelian group of coprime order [17], thus $\Gamma$ contains triangles in this case. So we can restrict ourselves to diameter 2 connected graphs without triangles of rank $\geq 4$. Suppose we have 3 coprime indices (i.e. 3 non-adjacent vertices in $\Gamma), a, b_{1}, b_{2}$. Then by Lemma 1 there exists a vertex $c$ which lies between $a$ and $b_{i}$. As we are assuming $\Gamma$ has no triangles and $c$ divides $a b_{i}$, this forces $c$ to have valency 2. Now consider $d\left(c, b_{j}\right)=2$, where $j \neq i, j=1,2$, this cannot be satisfied given our conditions and we have a contradiction. Thus, if $\Gamma$ has diameter 2 and no triangles, it cannot have 3 coprime vertices.

If we assume $\Gamma$ has at least 6 vertices, then either $\Gamma$ or its complement has a triangle [12, Theorem 2.2]. We have just shown that if we assume $\Gamma$ is of diameter 2 and has no triangles then also its complement has no triangles. Thus we can assume $\Gamma$ has less than 6 vertices. This leaves us with the cases when $\Gamma$ is a square or a pentagon. In these cases $G$ is an $F$-group, that is no non-trivial centraliser is contained in another non-trivial centraliser. Such groups have been classified by Rebmann [18]. A check of Rebmann's list gives no groups with graph $\Gamma$ a square or a pentagon. Thus, $G$ has rank at most 3.

\section{Solubility}

In this section we prove the following theorem. 
Theorem 2 Let $G$ be a finite group with the property that given any three distinct conjugacy class sizes greater than 1 there is a pair which is coprime. Then, $G$ is soluble.

We know from the results of Itô $[14,15]$ and A.Camina [7] that groups of rank less than 3 are soluble. So, by Theorem 1, we need only consider rank 3 groups.

If the group is an $F$-group we can see from Rebmann's list [18] that those that satisfy our criteria our soluble. Alternatively, note that in an $F$-group all centralisers are nilpotent, by Lemma 2, thus if we have two centralisers of coprime indices their product is $G$ and hence $G$ is soluble [13, VI.4.11, VI.4.14]. Thus for the rest of this section we will work under the following hypothesis.

Hypothesis Let $G$ be a finite group of conjugate rank 3 with conjugacy class sizes $\{1, a, b, c\}$ with $a$ divides $b$ and $\operatorname{gcd}(a, c)=1$. Further, there exist centralisers $A$ and $B$ with indices $a$ and $b$ respectively and $B<A$. Let $p$ be a prime number such that there exists a $p$-element with centraliser $A$.

To examine the structure of $A$ we need the following result, which is a combination of the results in [6] and [7] :-

Theorem 3 Let $G$ be a finite group, $p$ a prime number and $n$ an integer. Let $\pi$ be a non-empty set of primes. Assume that every $p^{\prime}$-element of $G$, for $p \in \pi$ has 1 or $m$ conjugates. Then $G$ is soluble. Furthermore,

- if $|\pi|>1, G$ is nilpotent and $m$ is a prime-power,

- if $\pi=\{p\}$ for some prime $p$ and $|G / Z(G)|$ is divisible by at least two primes different from $p$, then $G$ has -length $\leq 2$, q-length $=1, q$ a prime different from $p$ and $m$ is a power of $p$ or

- $\pi=\{p\}$ for some prime $p$ and $|G / Z(G)|=p^{e} q^{f}$ for some prime $q \neq p$ and natural numbers $e$ and $f$.

Next we state two simple, but useful, lemmas.

Lemma 2 [14, Proposition 1.1] Let $X$ be a minimal centraliser (i.e. properly contains no other centraliser) and let $\pi$ be the set of primes such that $p \in \pi$ if there exists a p-element $x$ with $C_{G}(x)=X$. Then

(i) if $|\pi|>1, X$ is abelian,

(ii) if $|\pi|=\{p\}, X=P_{0} \times U$ where $P_{0}$ is a p-group and $U \leq Z(X)$. 
Lemma 3 [7, Lemma 1] Let $p$ be a prime dividing the order of $G$. If $x \in$ $G$ and $\operatorname{Ind}_{G}(x)=\operatorname{Ind}_{G}(y)$ for some non-central p-element $y$, then $C_{G}(x)$ contains non-central p-elements.

The following proposition considers the case when we have a centraliser $C$, of index $c$, that is not minimal.

Proposition 1 If there exists a centraliser of index $c$ which is non minimal then $G$ is soluble.

Proof. There is a centraliser $A$ of index $a$ which contains a centraliser $B$ of index $b$ and centraliser $C$ of index $c$ which contains a centraliser of index $b$. So Theorem 3 applies to both $A$ and $C$ and we can choose either to suit our convenience.

$¿$ From the hypothesis of the proposition we have $a \mid b$ and $c \mid b$. Since $\operatorname{gcd}(a, c)=1$ we get $b=a c u$. If $u=1$ the result follows from $[3,4]$. So we may assume that $u>1$ and that not both $b / a$ and $b / c$ are prime powers.

So assume $b / a$ is not a prime power and let there be a $p$-element of index $a$. Thus from Theorem 3 we have $b / a=p^{e} q^{f}$ for distinct primes $p$ and $q$. So $b=a p^{e} q^{f}=a c u$, thus $c u=p^{e} q^{f}$ and $b / c=a u$. If $b / c$ is a prime-power then $a, c$ and $u$ are prime-powers and $G / Z(G)$ is a $\{p, q\}$-group and so is soluble.

We now consider the case when $b / c$ is not a prime-power. Let $x$ be chosen so that $\mathrm{C}_{G}(x)=C$ and $v$ chosen so that $\mathrm{C}_{G}(v)=D<C$ where $|D|=|B|$. Further $b / c$ is divisible by exactly two primes, say $b / c=r^{\alpha} s^{\beta}$ for primes $r$ and $s$. Since $u$ divides $b / c$ at least one of these primes is either $p$ or $q$. So $|\{p, q, r, s\}| \leq 3$. If $\{p, q, r, s\}=\{p, q\}$ then $G / Z(G)$ is a $\{p, q\}$-group and so is soluble.

Now suppose $|\{p, q, r, s\}|=3$ and $b / c=a u$ is divisible by a prime $r \neq p, q$ and $r \mid a$. Let $R<C$ be a Sylow $r$-subgroup of $G$. Either there is an $r$-element whose centraliser is $C$ or one whose centraliser, say $E$, has order $|B|$. But in either case there is a non-central $r$-element in $E$. Note that both $B$ and $E$ have the same order and both contain the centre of $G$, so the Sylow $r$ subgroup of $B$ has order greater than the Sylow $r$-subgroup of $Z(G)$ and hence $B$ contains a non-central $r$-element. From the conditions of Theorem 3 we know that $B$ contains no non-central $r$-element thus we have a contradiction.

We will consider the various possibilities for the set of non-trivial indices of $p^{\prime}$-elements, say $S$. So $S=\{a, b, c\},\{a, b\},\{a, c\},\{b, c\},\{a\},\{b\}$ or $\{c\}$. 
Lemma 4 Using the notation above if $|S|=1$ then $G$ is soluble.

Proof. This means that $G$ itself satisfies the hypotheses of Theorem 3 and thus is soluble.

For $p$-elements, let $T$ be the set of non-trivial indices of $p$-elements. We know $a \in T$ so $T=\{a, b, c\},\{a, b\},\{a, c\}$ or $\{a\}$.

Lemma 5 If there is a $p^{\prime}$-element, say $y$, of index a then $G$ is soluble.

Proof. Let $y$ be a $p^{\prime}$-element of index $a$ and let $\mathrm{C}_{G}(y)=A_{1}$ and note $\left|A_{1}\right|=|A|$, where $A=\mathrm{C}_{G}(x)$, and $x$ is a $p$-element. If $A_{1}=A$ then $A$ would be nilpotent by Theorem 3, we can assume $C$ is minimal by Proposition 1, and hence nilpotent, and thus $G$ is soluble as required. By Lemma 3 we have that $A_{1}$ contains a non-central $p$-element, say $u$. One possibility is that $\mathrm{C}_{G}(u)=A_{1}$ and then $A_{1}$ is nilpotent by Theorem 3. Again assuming that $C$ is minimal and hence nilpotent it follows that $G$ is soluble.

We now suppose that $\mathrm{C}_{G}(u) \neq A_{1}$ and there is a centraliser $B_{1}$ in $A_{1}$, we show that this cannot happen. If $b / a$ is a prime-power it is a power of $p$ by looking at $A$ but a different prime-power by looking at $A_{1}$ so this is false.

So $A$ and $A_{1}$ satisfy the third condition of Theorem 3 and look alike with $p$ and $q$ playing reverse roles. If $p$ does not divide $a$ then both $A$ and $A_{1}$ contain Sylow $p$-subgroups of $G$. Conjugating as necessary find a Sylow $p$ subgroup $P$ such that $P \leq A \cap A_{1}^{g}$. Recall $C_{G}(x)=A$ so $x \in Z(P)$ but then $x \in Z(G)$ because if not $\operatorname{Ind}_{A_{1}^{g}}(x)$ would be prime to $p$ contradicting the observation that $b / a$ is not a prime-power. So $p$ divides $a$. Similarly we show that $q$ divides $a$. However since $|G|=a|A|$ and $c$ and $a$ are coprime this gives that $c$ divides $|A|$, but $|A / Z(G)|$ is a $\{p, q\}$-group as we have that $b / a$ is not a prime-power and we are in the third case of Theorem 3 . Then $c$ is divisible by either $p$ or $q$ which contradicts $\operatorname{gcd}(a, c)=1$.

We now need to consider the case $S=\{b, c\}$.

Lemma 6 Assuming $C$ minimal there is no group such that $S=\{b, c\}$ and $c \in T$.

Proof. Let $u$ be a $p$-element so that $\mathrm{C}_{G}(u)=C$. Now there exists a noncentral $p^{\prime}$-element in $C$ by Lemma 3 and this cannot have index $b$ as we are assuming $C$ is minimal. So $C$ is abelian by Lemma 2. If $p$ does not divide $c$ then $C$ contains a Sylow $p$-subgroup of $G$ and so $C$ must contain a $p$-element 
of index $a$. If $p$ does not divide $a$ then any $p$-element of index $a$ is central in a Sylow $p$-subgroup of $G$ and so there is such an element which centralises $u$. Hence $C$ contains a $p$-element of index $a$, but this is a contradiction to the fact that $C$ is abelian.

Lemma 7 If $T \subseteq\{a, b\}$ and $b \in S$ then $G$ is soluble.

Proof. We begin by showing that $O_{p}(G)$ is not central. Consider the quotient $H=G / Z(G)$. This can be written as a product $H=(A / Z(G))(C / Z(G))$ where $A=C_{G}(x)$ is a centraliser of index $a$ and $C$ is a centraliser of index c. $A$ is soluble and $C$ is nilpotent since we are assuming $C$ is minimal (by Proposition 1). Since $x \notin Z(G)$ it follows that $A / Z(G)$ has a non-trivial centre. By Amberg and Kazarin [1], we get that $(Z(A) / Z(G))^{H}$ is an abelian normal subgroup of $H$. We see that $x$ is in the pre-image of $(Z(A) / Z(G))^{H}$. So $x \in O_{p}(G)$. Note that applying this to any $p$-element of index $a$ we see that every such element is in $O_{p}(G)$.

Let $P_{0}=O_{p}(G)$ and consider $K=C_{G}\left(P_{0}\right)$, note $K \neq G$, as $P_{0}$ contains non-central $p$-elements. We aim to show that $P_{0} K$ contains a Sylow $p$-subgroup of $G$. If there are no $p$-elements of index $b$ this is clear, so let $x$ be a $p$-element of index $b$. Now $C_{G}(x)$ contains a non-central $p^{\prime}$-element by Lemma 3, say $u$ and so $C_{G}(x)=C_{G}(u)=U \times V$, where $U$ is $p^{\prime}$-group and $V$ is a $p$-group with the same centraliser. An application of Thompson's Lemma $[11,5.3 .4]$ gives that $C_{G}(x) \leq K$. So we have $P_{0} K$ contains the Sylow $p$-subgroups of $G$.

The quotient $G / O_{p}(G) K$ is a product of the images of $A$ and $C$. By considering the three cases of Theorem 3 we see that both the image of $C$ and the image of $A$ are nilpotent. So $G / O_{p}(G) K$ is soluble.

To show that $G$ is soluble we need to show that $O_{p}(G) K$ is soluble. Note that $O_{p}(G)$ is soluble and that $K$ is soluble as the centraliser of any noncentral $p$-element is.

This final lemma completes the jigsaw.

Proof of the corollaries. As noted previously, groups of rank less than 3 are soluble $[14,15,7]$. So, we suppose $G$ has rank 3 and the non-trivial conjugacy class sizes are given by $\{a, b, c\}$. If there is a pair of conjugacy class sizes which are coprime then $G$ is soluble by the Main Theorem. Supposing no pair of conjugacy class sizes is coprime consider the case when there is a pair, say $a$ and $b$, such that $a$ divides $b$. Choose a prime $p$ which divides 
$\operatorname{gcd}(a, c)$, then $p$ also divides $b$. Thus the centre of a Sylow $p$-subgroup would be central. For Corollary A, this contradicts the hypothesis that $G$ has trivial centre. For Corollary B, this contradicts the hypothesis that $G$ is an $A$-group. So, in both corollaries we are left with the case that $G$ is an $F$-group, a check of Rebmann's list [18] yields the result.

\section{References}

[1] Bernhard Amberg and Lev Kazarin, On the product of a nilpotent group and a group with non-trivial center. J. Algebra, 311, (2007) 69-95.

[2] Elsa Fisman and Zvi Arad, A proof of Szep's conjecture on nonsimplicity of certain finite groups. J. Algebra, 108(2)(1987) 340-354.

[3] Antonio Beltrán and María José Felipe, Variations on a theorem by Alan Camina on conjugacy class sizes. J. Algebra, 296(1) (2006) 253-266.

[4] Antonio Beltrán and María José Felipe, Some class size conditions implying solvability of finite groups, J. Group Theory, 9(6) (2006) 787-797.

[5] Antonio Beltrán and María José Felipe, Structure of finite groups under certain arithmetical conditions on class sizes J. Algebra, 319(3) (2008) 897-910.

[6] A. R. Camina, Conjugacy classes of finite groups and some theorems of N. Itô. J. London Math. Soc. (2), 6 (1973) 421-426.

[7] A. R. Camina, Finite groups of conjugate rank 2. Nagoya Math. J., 53 (1974) 47-57.

[8] Rachel Camina, Schemes and the IP-graph J. Algebraic Comb, 28(3) (2008) 271-279.

[9] Edward A. Bertram, Marcel Herzog \& Avinoam Mann, On a graph related to conjugacy classes of groups, Bull. London Math. Soc. 22 (1990) $569-575$.

[10] Ming Fang \& Pu Zhang, Finite groups with graphs containing no triangles, J. Algebra 264 (2003), 613-619. 
[11] Daniel Gorenstein, Finite Groups, Harper and Row, 1968.

[12] F. Harary, Graph Theory, Addison-Wesley, 1969.

[13] B. Huppert, Endliche Gruppen I, Springer-Verlag, 1967.

[14] Norburo Itô, On finite groups with given conjugate types I, Nagoya Mathematical J. 6 (1953), 17-28.

[15] Noboru Itô, On finite groups with given conjugate types. II. Osaka J. Math. 7 (1970), 231-251.

[16] Noboru Itô, On finite groups with given conjugate types. III. Math. Z. 117 (1970), 267-271.

[17] L.S. Kazarin, On groups with isolated conjugacy classes. Izv. Vyssh. Uchebn. Zaved. Mat., 7 (1981), 40-45.

[18] Jochen Rebmann, F-Gruppen. Arch. Math. (Basel), 22 (1971) 225-230.

[19] Serge Tchounikhin, Simplicité du groupe fini et les ordres de ses classes d'éléments conjugués. Compt. Rend. Acad. Sci. (Paris) 191 (1930), 397-399. 